\title{
Rapidly progressive Bronchiolitis Obliterans Organising Pneumonia presenting with pneumothorax, persistent air leak, acute respiratory distress syndrome and multi-organ dysfunction: a case report Indranil Chakravorty*1, William LG Oldfield² and Carlos MH Gómez²
}

Address: ${ }^{1}$ Department of Respiratory Medicine, Lister Hospital, Beds \& Herts Postgraduate Medical School, Stevenage, UK and ${ }^{2}$ Adult Intensive Care Unit, St Mary's Hospital, London, UK

Email: Indranil Chakravorty* - i.chakravorty@herts.ac.uk; William LG Oldfield - William.Oldfield@St-Marys.nhs.uk; Carlos MH Gómez - Carlos.Gomez@St-Marys.nhs.uk

* Corresponding author

Published: 6 May 2008

Received: 15 June 2007

Journal of Medical Case Reports 2008, 2:145 doi:10.1 I86/1752-1947-2-145

Accepted: 6 May 2008

This article is available from: http://www.jmedicalcasereports.com/content/2/1//45

(C) 2008 Chakravorty et al; licensee BioMed Central Ltd.

This is an Open Access article distributed under the terms of the Creative Commons Attribution License (http://creativecommons.org/licenses/by/2.0), which permits unrestricted use, distribution, and reproduction in any medium, provided the original work is properly cited.

\begin{abstract}
Introduction: Bronchiolitis Obliterans Organising Pneumonia (BOOP) may often present initially as a recurrent spontaneous pneumothorax and then develop multi-system complications.

Case presentation: A 17-year-old boy presented with a pneumothorax, which developed into rapidly progressive Bronchiolitis Obliterans Organising Pneumonia (BOOP). He developed multiorgan dysfunction (including adult respiratory distress syndrome, oliguric renal failure, acute coronary syndrome, cardiac failure and a right atrial thrombus) which necessitated prolonged intensive care. Diagnosis was confirmed on open lung biopsy and he responded well to treatment with corticosteroids.

Conclusion: BOOP is exquisitely sensitive to oral corticosteroids but if the diagnosis is not considered in such patients and appropriate treatment instituted early, BOOP may often lead to prolonged hospital admission with considerable morbidity.
\end{abstract}

\section{Introduction}

Bronchiolitis Obliterans Organising Pneumonia (BOOP) is a clinico-pathological entity [1] characterised histologically by polypoid masses of granulation tissue in the lumen of small airways, alveolar ducts and alveoli coexisting with fibrosis [2]. Its distribution is patchy, with preservation of background architecture [1]. Lung function tests demonstrate impaired diffusion capacity with a restrictive picture [3] while the radiological appearance is of patchy consolidation and alveolar infiltrates in a peribronchiolar or pleural distribution [4]. We report on what we believe to be the first case of BOOP associated with persistent air leak, multiple organ failure and acute coronary syndrome.

\section{Case presentation}

A 17-year-old Asian man presented with a spontaneous pneumothorax, which was treated initially by needle aspiration. It recurred within a week with complete right-sided pneumothorax requiring water-sealed intercostal drainage. The air leak persisted after one week and the patient developed pyrexia with associated neutrophilia and raised serum inflammatory markers. A chest radiograph showed a hydro-pneumothorax and culture of the pleural fluid 
grew Pseudomonas aeruginosa and Methicillin-resistant Staphylococcus aureus (MRSA). He was treated with intravenous Piperacillin-Tazobactam resulting in a full clinicradiological recovery.

A week later his fever returned and repeat chest radiograph showed right middle and lower lobe consolidation associated with recurrent hydro-pneumothorax. A new intercostal drain was inserted and he was intubated and ventilated due to the rapid onset of severe respiratory distress. Computed tomogram (CT scan) of the chest confirmed bilateral patchy consolidation in association with widespread ground-glass opacities, sub-pleural cavitation and bilateral pleural effusions. He underwent an open thoracotomy, which did not reveal any macroscopic evidence of an organised or loculated empyema.

Postoperatively he developed multi-organ dysfunction with oliguric renal failure, prolonged mechanical ventilation, consumption coagulopathy, elevated aminotransaminases and an anteroseptal myocardial infarction with moderate left ventricular systolic dysfunction as evidenced by transoesophageal echocardiography (TOE) and raised troponin $\mathrm{I}$.

Multiple sampling of blood, urine and bronchoscopic lavage fluid for culture did not show any new bacterial, fungal or viral infection and the leukocyte count remained normal. Serological testing for atypical pneumonia (Legionella, Mycoplasma, Chlamydia and Coxiella), viral infection (Hepatitis virus $A, B$ \& $C$, Cytomegalovirus, Herpes viruses), and human immunodeficiency virus was negative. His autoimmune screen was negative (Anti-nuclear antibody, Rheumatoid factor and Anti-cytoplasmic antibodies).

He was treated with broad-spectrum antibiotics (Amoxycillin with Clavulinic acid, Clarithromycin, Piperacillin-Tazobactam, Gentamicin and Vancomycin) and an anti-fungal (Voriconazole) for four weeks without any clinical or radiological improvement.

He developed a right-sided broncho-pleural fistula complicated by pneumo-mediastinum and pneumo-pericardium, widespread consolidation, alveolar infiltrates, pulmonary haemorrhage and bilateral pleural effusions. He deteriorated again in his $4^{\text {th }}$ week with pyrexia and increasing inotrope dependence. A TOE confirmed persistent poor left ventricular function and a right atrial thrombus. He underwent median sternotomy and cardiopulmonary bypass to enable removal of the thrombus, at which time the opportunity was taken to perform an open lung biopsy from the right middle lobe. This showed changes showing alveolar exudates consistent with BOOP in association with patchy pulmonary haemorrhage and alveolar exudate.

He was commenced on corticosteroids (Prednisolone 1.5 $\mathrm{mg} / \mathrm{kg}$ ) with improvement clinically and radiologically within 72 hours. This was manifested by a reduction in oxygen requirement, reduced inflammatory markers, resolution of fever and disappearance of radiographic infiltrates.

Two weeks later, he was transferred to level II care for further weaning and rehabilitation. He represented three months later with a recurrent right-sided pneumothorax and underwent a pleurectomy. Subsequently, there have been no recurrences and the corticosteroid therapy has been rapidly weaned.

\section{Discussion}

BOOP may be idiopathic (which is associated with a better prognosis) [5] or secondary to bacterial (Mycoplasma) or viral infections (Human immunodeficiency virus, Herpes simplex virus), pharmacological agents (Nitrofurantoin, Sulfasalazine) [6], chemotherapy, radiotherapy [7] and connective tissue disorders.

Characteristic CT appearances in conjunction with broncho-alveolar lavage [8] finding of relative neutrophilia and reduction in the $\mathrm{CD} 4 / \mathrm{CD} 8$ ratio, is suggestive of BOOP. Transbronchial [8] and CT guided percutaneous approaches have a poor yield due to the patchy distribution of BOOP and small sample size. The differential diagnosis includes Acute Interstitial Pneumonitis and respiratory distress syndrome both of which may be associated with pneumonia or BOOP [9], as well as all the causes of cardiogenic pulmonary oedema. In patients who present with fulminant widespread consolidation with alveolar infiltrates, treatment is usually commenced empirically after collection of microbiological specimens.

However the potential benefit of timely corticosteroid therapy in patients with BOOP may justify the added risk of an open lung biopsy $[10,11]$ especially when obvious infective or cardiogenic causes cannot be identified. Patients with BOOP have a $>65 \%$ cure rate on corticosteroid therapy in most case series [1].

The initial sequence of events described in this case with a primary pneumothorax followed by a persisting air leak and signs of infection are not unknown. In our patient however, after an initial full clinico-radiological recovery, there was recurrence of the leak and development of a rapidly progressive 'acute inflammatory state' associated with multi-organ dysfunction, absence of infection and failure of broad-spectrum anti-microbials and an anti-fungal 
agent to improve clinical or radiological parameters. This was unusual and suggested an alternative pathology.

The second peculiarity of our case is the fulminant progression of BOOP into multi-organ failure after two weeks of apparent stability. A rapidly progressive type with multi-organ failure has indeed been described, albeit rarely [12]. Our patient, however, is to our knowledge the first reported case of cured or silent BOOP undergoing such conversion into a fulminant form.

This case highlights the importance of considering BOOP in the differential diagnosis of culture-negative respiratory failure in previously healthy patients. The 'Air Leak Syndrome' type of BOOP is associated with persisting broncho-pleural fistula, pneumo-mediastinum and pneumopericardium and has been reported only rarely [13-15].

\section{Conclusion}

To our knowledge the association in a previously healthy teenager of non-occlusive, Troponin I positive, acute coronary syndrome (with TOE confirmation of regional wall motion abnormalities but normal coronary arteries on CT angiogram as well as on direct surgical examination) with BOOP has not been hitherto described. In our patient appropriate treatment of the underlying left ventricular dysfunction did not alter the radiological appearances on serial high resolution CT scans. Although the likely explanation for the acute coronary syndrome in this patient may have been intravascular thrombosis there was no evidence of disseminated intravascular coagulation and, moreover, coagulation studies revealed raised prothrombin and thrombin times and thrombocytopenia with normal fibrinogen levels. The aetiology of acute coronary syndrome in association with BOOP remains unclear.

\section{Competing interests}

The authors declare that they have no competing interests.

\section{Authors' contributions}

All three authors were fully involved with the management of this case while in hospital and have equally contributed to the design, drafting and editing of the article submitted. The authors also acknowledge the contribution of other colleagues in the management of this challenging case.

\section{Consent}

Written informed consent was obtained from the patient for publication of this case report and accompanying images.

\section{Acknowledgements}

We wish to acknowledge the important contribution to the management of this patient made by our colleagues from Intensive Care Medicine, Res- piratory Medicine, Cardiology, Radiology, Cardiothoracic Surgery and by the nurses in Intensive Care and High Dependency wards. Written informed consent has been obtained. All 3 authors were heavily involved managing, researching and preparing the manuscript. There are no conflicts of interest to declare.

\section{References}

I. Epler GR, Colby TV, McLoud TC, Carrington CB, Gaensler EA: Bronchiolitis obliterans organizing pneumonia. N Engl J Med 3 I 2(3): 152-8. 1985 Jan 17

2. Myers JL, Katzenstein AL: Ultrastructural evidence of alveolar epithelial injury in idiopathic bronchiolitis obliterans-organizing pneumonia. Am J Pathol 1988, I32(I): 102-9.

3. Muller NL, Guerry-Force ML, Staples CA, Wright JL, Wiggs B, Coppin $C$, Pare P, Hogg JC: Differential diagnosis of bronchiolitis obliterans with organizing pneumonia and usual interstitial pneumonia: clinical, functional, and radiologic findings. Radiology 1987, 162(I Pt I): I5I-6.

4. Muller NL, Staples CA, Miller RR: Bronchiolitis obliterans organizing pneumonia: CT features in 14 patients. AJR Am J Roentgenol 1990, I54(5):983-7.

5. Lohr RH, Boland BJ, Douglas WW, Dockrell DH, Colby TV, Swensen SJ, Wollan PC, Silverstein MD: Organizing pneumonia. Features and prognosis of cryptogenic, secondary, and focal variants. Arch Intern Med I 57( I 2): I 323-9. 1997 Jun 23

6. Epler GR: Drug-induced bronchiolitis obliterans organizing pneumonia. Clin Chest Med 2004, 25(I):89-94.

7. Mokhtari M, Bach PB, Tietjen PA, Stover DE: Bronchiolitis obliterans organizing pneumonia in cancer: a case series. Respir Med 2002, 96(4):280-6.

8. Poletti V, Cazzato S, Minicuci N, Zompatori M, Burzi M, Schiattone ML: The diagnostic value of bronchoalveolar lavage and transbronchial lung biopsy in cryptogenic organizing pneumonia. Eur Respir J 1996, 9(12):25 I3-6.

9. Perez de Llano LA, Soilan JL, Garcia Pais MJ, Mata I, Moreda M, Laserna B: Idiopathic bronchiolitis obliterans with organizing pneumonia presenting with adult respiratory distress syndrome. Respir Med 1998, 92(6):884-6.

10. Patel SR, Karmpaliotis D, Ayas NT, Mark EJ, Wain J, Thompson BT, Malhotra A: The role of open-lung biopsy in ARDS. Chest 2004, I 25(I): 197-202.

II. Kramer MR, Berkman N, Mintz B, Godfrey S, Saute M, Amir G: The role of open lung biopsy in the management and outcome of patients with diffuse lung disease. Ann Thorac Surg 1998, 65(I): 198-202.

12. Kofteridis DP, Bouros DE, Vamvakas LN, Stefanaki KS, Voludaki AE, Barbounakis EM, Emmanouel DS: Pneumothorax complicating fatal bronchiolitis obliterans organizing pneumonia. Respiration 1999, 66(3):266-8.

13. Iwanaga T, Hirota T, Ikeda T: Air leak syndrome as one of the manifestations of bronchiolitis obliterans organizing pneumonia. Intern Med 2000, 39(2): 163-5.

14. Yang DG, Kim KD, Shin DH, Choe KO, Kim SK, Lee WY: Idiopathic bronchiolitis obliterans with organizing pneumonia presenting with spontaneous hydropneumothorax and solitary pulmonary nodule. Respirology 1999, 4(3):267-70.

15. Nizami IY, Kissner DG, Visscher DW, Dubaybo BA: Idiopathic bronchiolitis obliterans with organizing pneumonia. An acute and life-threatening syndrome. Chest 1995, I08(I):27|-7. 